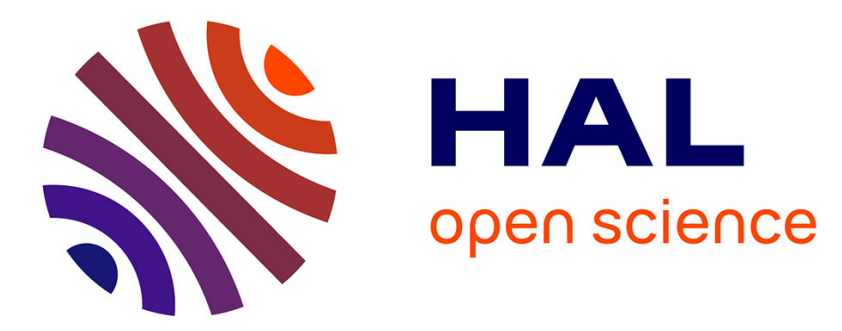

\title{
Stochastic maximum allowable transmission intervals for the stability of linear wireless networked control systems
}

\author{
Vineeth Varma, Romain Postoyan, Irinel-Constantin Morarescu, Jamal
}

Daafouz

\section{- To cite this version:}

Vineeth Varma, Romain Postoyan, Irinel-Constantin Morarescu, Jamal Daafouz. Stochastic maximum allowable transmission intervals for the stability of linear wireless networked control systems. 56th IEEE Conference on Decision and Control, CDC 2017, Dec 2017, Melbourne, Australia. hal-01652498

\section{HAL Id: hal-01652498 \\ https://hal.science/hal-01652498}

Submitted on 26 Mar 2018

HAL is a multi-disciplinary open access archive for the deposit and dissemination of scientific research documents, whether they are published or not. The documents may come from teaching and research institutions in France or abroad, or from public or private research centers.
L'archive ouverte pluridisciplinaire HAL, est destinée au dépôt et à la diffusion de documents scientifiques de niveau recherche, publiés ou non, émanant des établissements d'enseignement et de recherche français ou étrangers, des laboratoires publics ou privés. 


\title{
Stochastic maximum allowable transmission intervals for the stability of linear wireless networked control systems
}

\author{
Vineeth S. Varma, Romain Postoyan, Irinel-Constantin Morărescu and Jamal Daafouz
}

\begin{abstract}
We investigate the scenario where a plant, modeled as a linear deterministic discrete-time system, is controlled through a wireless communication network. The controller is designed by emulation, meaning that we construct it to stabilize the origin of the plant while ignoring communication constraints and then implement the control law over the network. The transmissions over the wireless channel are time-varying and uncertain, in particular, the probability of successful communication (i.e., no packet is dropped) between the plant and the controller depends on the resources utilized, such as the allocated bandwidth or the transmission signal power. As a result, we provide conditions on the varying intertransmission times to ensure the mean square stability of the closed-loop system. The novelty is that stability properties are characterized by not only the length of the inter-transmission interval (often called the MATI in the deterministic literature), but also by the probability of successful communication before and after this interval, referred to as the stochastic allowable transmission interval (SATI). These conditions could then be utilized by radio engineers/researchers to design energy-efficient communication strategies while ensuring the mean square stability of the control system.
\end{abstract}

\section{INTRODUCTION}

Wireless networks offer appealing features for the implementation of control loops, as these allow remote control and exhibits many advantages over traditional wired pointto-point set-ups in terms of flexibility, ease of maintenance, reduced weight and volume. On the other hand, the communication constraints induced by the network, such as packet drop-outs, scheduling, etc., need to be appropriately handled in the design of the control law to guarantee the desired requirement for the closed-loop system, called in this context, a networked control system (NCS); see [7], [8], [21] and the references therein. A key parameter of NCS is the maximum allowable transmission interval (MATI), which is a bound on the maximum time between two successive transmissions. Numerous works address the construction of computable bounds on the MATI under which the control requirements are ensured, see [1], [4], [9], [11]-[13] for stability results, [14] for observers design and [15] for tracking control. The MATI is a deterministic constraint, as two successive transmission times are not allowed to be spaced by more than this quantity. This requirement may be difficult to achieve when working with wireless NCS (WNCS), for which transmissions are often modeled as a stochastic process. While

Vineeth S. Varma, Romain Postoyan, Irinel-Constantin Morărescu and Jamal Daafouz are with the Université de Lorraine, CRAN, UMR 7039 and the CNRS, CRAN, UMR 7039, France, vineeth. satheeskumar-varma@univ-lorraine.fr. This work was supported by the ANR project Computation Aware Control Systems, ANR-13-BS03-004-02. relaxations of the MATI to cope with packet drops or delays are available in e.g., [2], [4], [6], [11], these results still have a strong deterministic flavor, which may not be suitable for WNCS. A stochastic alternative to the notion of MATI is therefore needed when dealing with WNCS: this is the main purpose of this study.

A first observation is that we can no longer characterize stability exclusively based on the length of the intertransmission interval, $N$, in the stochastic setting. Indeed, we also need to take into account the (cumulative) probability that a transmission occurs within these $N$ steps, which we denote $\eta$. The value $\eta$ depends on the probability of successful transmission at each time step in the interval of $N$. We also need to consider what happens if the time since the last successful transmission becomes bigger than $N$. In this case, we want to maximize the chance for a packet to be successfully transmitted. We therefore assume that every transmission after $N$ steps, occurs with probability $\delta$. The parameter $\delta$ represents the physical limitations of the communication system in terms of packet success [18], and it is impossible to achieve a higher communication success probability than $\delta$ at any time instant regardless of the resources utilized, see also [17], [22] for practical details. In other words, when the inter-transmission interval is larger than $N$, transmission is repeatedly attempted with all the available resources, i.e., with a success probability of $\delta$, until the transmission is successful. As a result, stability now depends on $N, \eta$ and $\delta$ and we term this ensuing notion, the $(\eta, \delta)$-stochastic maximum allowable transmission interval, $(\delta, \eta)$-SATI in short. The $(\eta, \delta)$-SATI is not only useful to investigate stability of WNCS, it is also relevant to optimize the transmission energy. Indeed, we have shown in [19] how to optimize the energy used to communicate in WNCS with stochastic channel fading, while guaranteeing a MATI condition. Because of the latter, we had to make strong assumptions on the channel state, in particular we assumed that a successful transmission is possible at any given time. These assumptions can be relaxed when imposing a $(\eta, \delta)$-SATI constraint on the network instead; this will be investigated in future work.

Before we explain in more detail the content of this study, we need to point out that related results are available in the literature. In particular in [3], [10], [20], stochastic timevarying transmission times are considered and stability is established under conditions on the i.i.d probability distributions of the inter-transmission intervals. In Section V of [10], two cases are studied, when the inter-transmission intervals follow a given i.i.d distribution, or when the inter- 
transmission intervals are a Markov process, with each mode of the associated Markov jump system denoting a certain inter-transmission interval. Our results are significantly different from these works as we characterize stability depending on the inter-transmission interval length, $N$, the cumulative probability $\eta$ that a transmission occurs before $N$ steps have elapsed, and $\delta$, instead of the probability distribution of the inter-transmissions intervals. This major difference prevents us from applying the results in [3], [10] and requires developing a new framework.

In this paper, we consider plants modeled by a linear timeinvariant discrete-time system. The controller is designed by emulation, meaning that we synthesize it while ignoring the network. We allow for dynamic output-feedback laws, hence covering static feedback stabilizers as a particular case. We then take into account the stochastic communication constraint induced by the network. We concentrate on the impact of time-varying successful transmission times and we ignore possible transmission delays and quantization effects. Various holding strategies are allowed when implementing the controller, such as zero-order hold, zeroing [16], or model-based techniques [10]. The overall system is modeled as a Markov jump linear systems like in Section V of [10], but with each mode of the Markov jump system representing the time since the last transmission instant, and not the inter-transmission interval as done in [10]. Note that intertransmission intervals measure the time interval between two successful transmissions. Our objective is to ensure the mean square stability (MSS) of this Markov jump linear system depending on the values of $\eta, \delta$ and $N$ (and not the probabilities of having certain inter-transmission intervals). Sufficient conditions in terms of matrix inequalities are provided, and then extended to the case where $\eta$ and $\delta$ are time-varying. The results are then illustrated on a numerical example.

The paper is organized as follows. The problem is stated in Section II. In Section III, we formally define the SATI and we model the WNCS as a Markov jump linear system in Section IV. In Section V, we provide conditions in the form of linear matrix inequalities (LMIs) under which the WNCS is MSS. Finally, in Section VI, some numerical results are presented, which highlight the relevance of our approach.

Notation. Let $\mathbb{R}:=(-\infty, \infty), \mathbb{R}_{\geq 0}:=[0, \infty)$, and $\mathbb{Z}_{\geq 0}:=$ $\{0,1,2, \ldots\}$. We use $E$ for the expectation taken over the relevant stochastic variables and $\mathbf{1}(\cdot)$ for the indicator function, taking the value 1 when the condition is satisfied and 0 otherwise.

\section{PRoblem StATEMEnT}

We consider the discrete-time linear system

$$
\begin{aligned}
x_{p}(t+1) & =A_{p} x_{p}(t)+B_{p} u(t) \\
y(t) & =C_{p} x_{p}(t),
\end{aligned}
$$

where $t \in \mathbb{Z}_{\geq 0}$ is the time, $x_{p}(t) \in \mathbb{R}^{n_{x}}$ is the plant state, $u(t) \in \mathbb{R}^{n_{u}}$ is the control input, $y(t) \in \mathbb{R}^{n_{y}}$ is the output and $n_{x}, n_{u}, n_{y} \in \mathbb{Z}_{\geq 0}$. The pairs $\left(A_{p}, B_{p}\right)$ and $\left(A_{p}, C_{p}\right)$ are assumed to be stabilizable and detectable, respectively.

We proceed by emulation and we first construct a stabilizing controller for system (1) given by

$$
\begin{aligned}
x_{c}(t+1) & =A_{c} x_{c}(t)+B_{c} y(t) \\
u(t) & =C_{c} x_{c}(t)+D_{c} y(t),
\end{aligned}
$$

where $x_{c}(t) \in \mathbb{R}^{n_{x}}$ is the controller state. A stabilizing controller of the form of (2) for system (1) always exists since $\left(A_{p}, B_{p}\right)$ is stabilizable and $\left(A_{p}, C_{p}\right)$ is detectable.

We are intested in the scenario where plant (1) and controller (2) communicate over a wireless channel. In particular, we investigate the case where the wireless network is between the sensors and the controller as seen in Figure 1a, or is between the controller and the actuator as seen in Figure 1b. As a result, the feedback loop is no longer closed at every time instant $t \in \mathbb{Z}_{\geq 0}$, but only at the instants $t_{i}, i \in \mathcal{I} \subseteq \mathbb{Z}_{\geq 0}$ when communication is successful.

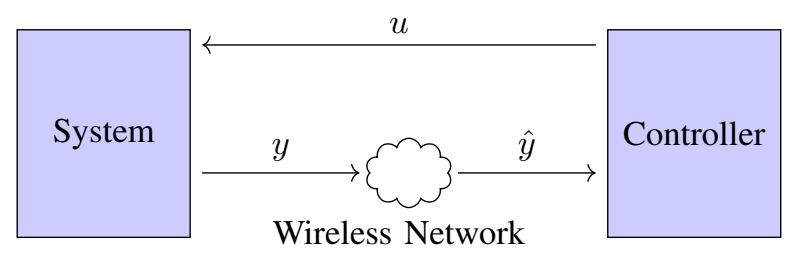

(a) Network between the sensors and the controller.

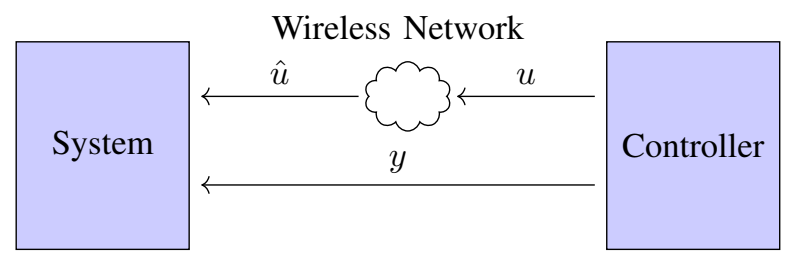

(b) Network between the controller and the actuators.

Fig. 1: Schematic of the networked control system.

When the WNCS is of the first case as in Figure 1a, (1) remains unchanged, but (2) becomes

$$
\begin{aligned}
x_{c}(t+1) & =A_{c} x_{c}(t)+B_{c} \hat{y}(t) \\
u(t) & =C_{c} x_{c}(t)+D_{c} \hat{y}(t),
\end{aligned}
$$

where $\hat{y}(t)$ denotes the networked version of the measurement available at the controller. When the network is between the controller and the actuators as in Figure 1b, (2) remains unchanged, but (1) becomes

$$
\begin{aligned}
x_{p}(t+1) & =A_{p} x(t)+B_{p} \hat{u}(t) \\
y(t) & =C_{p} x_{p}(t)
\end{aligned}
$$

where $\hat{u}(t)$ denotes the networked version of the control available at the actuator. In the following, we focus on the case where the network is between the sensors and the controller as in Figure 1a. The other case can be similarly handled and results in the same general model as shown in Section IV. Between two successive successful transmission 
instants, $\hat{y}(t)$ is held using the following holding function

$$
\left.\left.\hat{y}(t)=C_{g} \hat{y}(t-1)\right)+D_{g} x_{c}(t)\right)
$$

when $t \in \mathbb{Z}_{\geq 0}$ and $t \notin\left\{t_{i}\right\}_{i \in \mathcal{I}}$. For instance, the use of zero-order-hold devices leads to $\left(C_{g}, D_{g}\right)=(I, 0)$, and the use of zeroing strategies gives $\left(C_{g}, D_{g}\right)=(0,0)$. When the $x_{c}$-system in (2) is an observer, we can use a model-based holding strategy like in [10], which leads to $\left(C_{g}, D_{g}\right)=$ $\left(C_{p} B_{p} D_{c}, C_{p}\left(A_{p}+B_{p} C_{c}\right)\right)$. At $t_{i}, i \in \mathcal{I}$, we have

$$
\hat{y}\left(t_{i}\right)=y\left(t_{i}\right) .
$$

We now need to describe the sequence of successful transmission instants $t_{i}, i \in \mathcal{I}$. When the transmissions between the plant and the controller must satisfy a deterministic constraint, a key parameter is the MATI as we mentioned in the introduction. As we consider discrete dynamics, the MATI is the maximum interval $N \in \mathbb{Z}_{\geq 0}$ such that the stability of the original closed loop system is preserved, provided

$$
t_{i+1}-t_{i} \leq N
$$

for all $i \in \mathcal{I}$, see [13]. In this paper, we consider wireless networks, for which transmissions are typically modeled as a stochastic process. As a result, to demand that successful transmission occurs within the MATI may not be appropriate. We therefore need a new notion to ensure stability (in a stochastic sense), which not only depends on the length of the inter-transmission intervals as in (6), but also on the probability that a successful transmission occurs before a given time and that after this time has passed. We call this new notion $(\eta, \delta)$-SATI, where $\eta$ is the cumulative probability that communication was successful within the $(\eta, \delta)$-SATI, and $\delta$ is the maximum transmission success rate possible for the communication network at any given time after the interval has passed.

Our objective is to provide conditions on $\eta, \delta$ and the induced SATI such that the closed-loop networked control system is MSS. Before we study the stability properties, we will establish the technical framework for defining the $(\eta, \delta)$ SATI in the next section.

\section{STOCHASTIC ALLOWABLE TRANSMISSION INTERVAL}

The stability of the WNCS (4) depends on the sequence of successful communication instants $t_{i}, i \in \mathcal{I}$. To model the latter, we introduce the clock $\tau(t) \in \mathbb{Z}_{\geq 0}$ which counts the number of time instants since the last successful transmission

$$
\tau(t+1)=\left\{\begin{array}{c}
1 \\
\quad \text { when communication is successful at } t \\
\tau(t)+1 \\
\text { when no or failed communication at } t .
\end{array}\right.
$$

Let $N$ be a given transmission interval length. We consider that the probability that communication is successful is given by $f(\tau(t)) \in[0, \delta]$ for all $\tau(t) \leq N$. That is, communication success is a Bernoulli process, with the time since the last transmission triggering different communication modes. We allow $f$ to depend on $\tau(t)$, and on $t$ in the time-varying extension described in the remark below, as this probability depends on several factors like the time-varying wireless channel quality, transmission power etc. The sensor can know if a transmission was successful via a simple acknowledgement scheme, which is commonly used in wireless communication systems. The probability $\eta$ to transmit within this interval $N$ is therefore defined as

$$
\eta:=1-\prod_{i=1}^{N}(1-f(i)) .
$$

To be precise, we impose a constraint on the probability of the inter-transmission interval being larger than $N$, and $\eta$ denotes the probability that the inter-transmission interval was within the interval $N$. The motivation for characterizing communication policies in this manner is to decouple the control requirements and the communication aspects. As long as the inter-transmission interval $\tau(t)$ is within the communication interval $N$, i.e. $\tau(t) \leq N$, communication can be attempted with a lower resource consumption, like radio-transmit power or bandwidth, while ensuring that (8) is satisfied to have stability.

Recall that $\delta$ is the maximum success probability as explained in the above section, and so $f(\tau(t)) \leq \delta$ for all $t \in \mathbb{Z}_{\geq 0}$. We study a specific class of policies defined by the interval $N \in \mathbb{Z}_{\geq 0}$, after which communication is repeatedly attempted with all the available resources. That is, $f(\tau(t))=$ $\delta$ for all $\tau(t)>N$. If the inter-transmission interval is too large, i.e. more than $N$, all the available resources must be utilized, resulting in the maximum communication success rate of $\delta$.

A special case of interest is when $\eta=0$, i.e., no communication is attempted until $\tau(t)>N$. This quantity is highly relevant in practice as many communication systems, like remote sensors, have low computational capabilities and can only operate in two modes ON or OFF. Additionally, communication constraints do not allow updates in every time slot due to scheduling conflicts etc. As $\delta$ can be determined from the network settings, it becomes very easy to design a scheme that after a successful communication, waits for the duration $(0, \delta)$-SATI and then repeatedly attempts to send its message until it is received and so on. Therefore, we will refer to the $(0, \delta)$-SATI as simply the $\delta$-SATI.

Remark 1: We can easily extend our results when the communication probabilities are time-varying. That is, if the communication success at each time is given by $f(\tau(t), t) \in$ $[0, \delta(t)]$, such that $\delta(t) \in\left[\delta_{\min }, \delta_{\max }\right]$ with $0 \leq \delta_{\min } \leq$ $\delta_{\max } \leq 1$. This model is suitable when the wireless communication system has time-varying parameters. For instance, the quality of the wireless communications, commonly known as the channel fading, is a time-varying parameter, which determines the packet success rate. In such cases, instead of $\eta$, we will have $\eta(t)$ for each $t$ which we can constraint to lie in a certain bound, i.e. $0 \leq \eta_{\min } \leq \eta(t) \leq$ $\eta_{\max } \leq 1$ for all $t \in \mathbb{Z}_{\geq 0}$. 


\section{THE WNCS AS A MARKOV JUMP LINEAR SYSTEM}

In this section, we model the WNCS as a Markov jump linear system in order to then proceed with the stability analysis in Section V. As mentioned in Section III, we focus on the scenario where the network is between the sensors and the controllers as in Figure 1a; similar derivations apply when the network is between the controller and the actuators.

We define the concatenation of all the state variables as

$$
\chi(t):=\left(\begin{array}{c}
x_{p}(t) \\
x_{c}(t) \\
\hat{y}(t-1)
\end{array}\right)
$$

with $\hat{y}(-1)$ being chosen arbitrarily from $\mathbb{R}^{n_{y}}$. We thus write the overall model as

$$
\chi(t+1)=\left\{\begin{array}{l}
\mathcal{A}_{1} \chi(t) \\
\mathcal{A}_{2} \chi(t) \text { when communication is successful } \\
\text { when no or failed communication, }
\end{array}\right.
$$

with

$$
\mathcal{A}_{1}:=\left(\begin{array}{ccc}
A_{p}+B_{p} D_{c} C_{p} & B_{p} C_{c} & 0 \\
B_{c} C_{p} & A_{c} & 0 \\
C_{p} & 0 & 0
\end{array}\right)
$$

and

$$
\mathcal{A}_{2}:=\left(\begin{array}{ccc}
A_{p} & B_{p} C_{c} & B_{p} D_{c} \\
0 & A_{c} & B_{c} \\
0 & C_{g} & D_{g}
\end{array}\right) .
$$

System (10) is a linear jump system with two modes, depending on whether a successful transmission occurs at time $t \in \mathbb{Z}_{>0}$. To write the system as a Markov jump linear system, we introduce $N+1$ virtual modes as the transition probabilities are $f(\tau(t))$ for $\tau(t) \leq N$ and is a constant $\delta$ otherwise.

When communication is successful, i.e. $\tau(t+1)=1, \chi(t+$ $1)=\mathcal{A}_{1} \chi(t)$ and $\chi(t+1)=\mathcal{A}_{2} \chi(t)$ otherwise. Therefore, we model the dynamical system as a linear jump system with 2 distinct dynamics, but $N+1$ virtual modes (the dynamics are identical for $N$ of these modes) as shown in Figure 2.

The mode index $r(t) \in\{1,2, \ldots, N+1\}$ defined as $r(t)=$ $\tau(t)$ when $\tau(t) \leq N$ and $r(t)=N+1$ when $\tau(t)>N$. The associated jump probability matrix is $\Pi$ given by

$$
\Pi:=\left(\begin{array}{ccccc}
f(1) & f(2) & \ldots & f(N) & \delta \\
1-f(1) & 0 & \ldots & 0 & 0 \\
0 & 1-f(2) & \ldots & 0 & 0 \\
\vdots & & & & \\
0 & 0 & \ldots & 1-f(N) & 1-\delta
\end{array}\right)
$$

which can be deduced by studying Figure 2. There is a probability to jump to mode 1 , i.e., to communicate, given by $f(\tau(t))$ or equivalently $f(r(t))$ from any mode $r(t)$. The only other mode that can be reached from $r(t)$ is one with $\tau(t+1)=\tau(t)+1$, resulting in 0 probability of jumps to all the other states. Finally, it is also possible to jump from $r(t)=N+1$ to $r(t+1)=N+1$ as $r(t)=N+1$ represents any $\tau(t)>N$. As a result, the dynamics (10) are rewritten

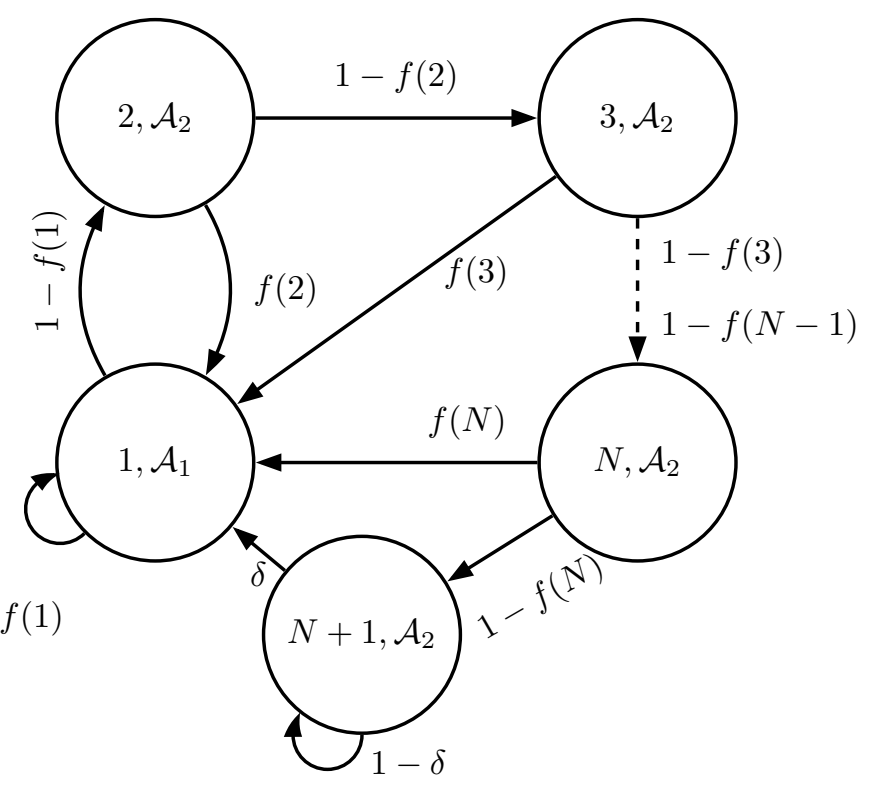

Fig. 2: The Markov chain with the modes representing the states

as

$$
\begin{aligned}
\chi(t+1) & =\mathcal{A}_{1+\mathbf{1}(r(t)>1) \chi(t)} \\
\operatorname{Pr}(r(t+1)=j \mid r(t)=i) & =\Pi_{i j}, \quad 1 \leq i, j \leq N+1,
\end{aligned}
$$

with $r(t)$ denoting the discrete-time process determining the mode of the Markov jump system.

With system (14) at hand, we are ready to proceed with the stability analysis in the next section.

Remark 2: When the wireless network is between the controller and the actuators as in $1 \mathrm{~b}$, we have

$$
\chi(t):=\left(\begin{array}{c}
x_{p}(t) \\
x_{c}(t) \\
\hat{u}(t-1)
\end{array}\right)
$$

with

$$
\mathcal{A}_{1}=\left(\begin{array}{ccc}
A_{p}+B_{p} D_{c} C_{p} & B_{p} C_{c} & 0 \\
B_{c} C_{p} & A_{c} & 0 \\
D_{c} C_{p} & C_{c} & 0
\end{array}\right),
$$

and

$$
\mathcal{A}_{2}=\left(\begin{array}{ccc}
A_{p} & 0 & B_{p} \\
B_{c} C_{p} & A_{c} & 0 \\
0 & C_{g} & D_{g}
\end{array}\right)
$$

\section{MAin RESUlTS}

Our goal is to determine conditions such that the system (14) is MSS defined as follows.

Definition 1: Given $N \in \mathbb{Z}_{\geq 0}, \eta, \delta \in[0,1]$, the system (14) is MSS if and only if for some $\beta \geq 1,0<\zeta<1$ and any $\chi(0) \in \mathbb{R}^{n_{x}} \times \mathbb{R}^{n_{x}} \times \mathbb{R}^{n_{y}}$,

$$
E\left[\chi(t)^{T} \chi(t)\right] \leq \beta \zeta^{t} \chi(0)^{T} \chi(0)
$$

We first derive the next lemma, which directly follows from the results in Section 4 of [5]. 
Lemma 1: System (14) is MSS if and only if there exists symmetric positive definite matrices $Q_{m}$, with $m \in$ $\{1,2, \ldots, N+1\}$, such that the following holds

$$
\mathcal{A}_{1+\mathbf{1}(m>1)}\left(\sum_{i=1}^{N+1} \Pi_{i, m} Q_{i}\right) \mathcal{A}_{1+\mathbf{1}(m>1)}^{T}<Q_{m}
$$

for all $1 \leq m \leq N+1$.

Lemma 1 is informative but it does not suit our purpose. Indeed, stability conditions are provided, but these involve the probabilities $f(\tau(t))$ through $\Pi$, see (13) and (14). We are looking for conditions, which are independent of $f(\tau(t))$, and only depend on $N, \eta$ and $\delta$. Therefore, we propose the next theorem in order to characterize MSS of (14) based on $\eta, \delta$ and $N$, allowing us to compute bounds on the $(\eta, \delta)$ SATI.

Theorem 1: Given $N \in \mathbb{Z}_{\geq 0}, \eta \in[0,1]$ and $\delta \in[0,1]$, the system (14) is MSS if there exists symmetric positive definite matrices $Q_{1}, Q_{N+1}$ and $S$ such that

$$
\begin{gathered}
\mathcal{A}_{1}\left(\delta Q_{N+1}+\eta S\right) \mathcal{A}_{1}^{T}<Q_{1} \\
\mathcal{A}_{2}\left((1-\delta) Q_{N+1}+(1-\eta) S\right) \mathcal{A}_{2}^{T}<Q_{N+1}
\end{gathered}
$$

and

$$
\mathcal{A}_{2}^{i} Q_{1}\left(\mathcal{A}_{2}^{i}\right)^{T}<S \quad \forall i \in\{0, \ldots, N-1\}
$$

holds.

Theorem 1 provides sufficient conditions for the system (14) to be MSS. These conditions can be easily verified and are dependent only on $N, \delta$ and $\eta$, and are independent of the probabilities $f(\tau(t))$ used during the flexible period. As $\delta$ is usually given from communication constraints, we have flexibility in choosing $N$ and $\eta$. For a given $N$ and $\delta$, as $0 \leq \eta \leq 1$, we can do a bisection based linear search to find the minimum $\eta$ required for having MSS. On the other hand, $N$ belongs to a discrete set, hence, for a given $(\eta, \delta)$, we can also do a line search to find the largest $N$ satisfying the LMIs, thereby obtaining a lower bound on the $(\eta, \delta)$-SATI. Note that when $\eta=1$ and $\delta=1$, a SATI always exists as the system (1)-(2) has its origin globally exponentially stable.

When $\eta=0$, the largest $N$ satisfying the matrix inequalities in Theorem 1 corresponds to the $\delta$-SATI. In this case, when $\eta=0$, we have less conservative matrix inequalities as seen from next corollary.

Corollary 1: Given $N \in \mathbb{Z}_{\geq 0}, \eta=0$ and $\delta \in[0,1]$, the system (14) is MSS if there exists symmetric positive definite matrices $Q_{1}$ and $Q_{N+1}$ such that

$$
\begin{gathered}
\delta \mathcal{A}_{1} Q_{N+1} \mathcal{A}_{1}^{T}<Q_{1} \\
\mathcal{A}_{2}\left((1-\delta) Q_{N+1}+\mathcal{A}_{2}^{N-1} Q_{1}\left(\mathcal{A}_{2}^{N-1}\right)^{T}\right) \mathcal{A}_{2}^{T}<Q_{N+1}
\end{gathered}
$$

holds.

As mentioned in Remark 1, our results can be easily extended to the case where $\eta$ and $\delta$ are time-varying. When the communication success at each time is given by $f(\tau(t), t) \in[0, \delta(t)]$ such that $\delta(t) \in\left[\delta_{\min }, \delta_{\max }\right]$ and $\eta_{\min } \leq \eta(t) \leq \eta_{\max }$ for all $t \in \mathbb{Z}_{\geq 0}$, we have the next result, whose proof follows similar lines as the proof of Theorem 1, and is therefore omitted. Here, the process is time-varying, but the probabilities for packet loss is bounded by the values $\delta_{\min }$ and $\delta_{\max }$. It might not be feasible to impose a timeinvariant constraint as in (8), and so we consider a timevarying $\eta(t)$ as well, which lies in the interval defined by $\eta_{\min }$ and $\eta_{\max }$.

Corollary 2: System (14) is MSS for a given interval $N$, $\eta_{\min } \leq \eta(t) \leq \eta_{\max }$ and $\delta(t) \in\left[\delta_{\min }, \delta_{\max }\right]$, if the following matrix inequalities are satisfied:

$$
\begin{gathered}
\mathcal{A}_{1}\left(\delta_{\max } Q_{N+1}+\eta_{\max } S\right) \mathcal{A}_{1}^{T}<Q_{1} \\
\mathcal{A}_{2}\left(\left(1-\delta_{\min }\right) Q_{N+1}+\left(1-\eta_{\min }\right) S\right) \mathcal{A}_{2}^{T}<Q_{N+1}
\end{gathered}
$$

and

$$
\mathcal{A}_{2}^{i} Q_{1}\left(\mathcal{A}_{2}^{i}\right)^{T}<S \quad \forall i \in\{0, \ldots, N-1\}
$$

for some $Q_{1}=Q_{1}^{T}>0, Q_{N+1}=Q_{N+1}^{T}>0$ and $S=$ $S^{T}>0$.

\section{NumERICAL RESUlts}

In this section, we apply our results on system (1) with $A_{p}=\left(\begin{array}{cc}1 & 0.1 \\ 0 & 1\end{array}\right), B_{p}=\left(\begin{array}{l}0 \\ 1\end{array}\right)$ and $C_{p}=I$, for which we design the controller (2) with $D_{c}=\left(\begin{array}{ll}-0.012 & -0.07\end{array}\right)$, $B_{c}=I$ and $C_{c}=A_{c}=0$. We then implement this controller over a network. For ease of exposition, we focus on the case where the probability of successful transmission only depends on the clock $\tau(t)$ and not explicitly on the time. We then apply Theorem 1 to study the impact of $N, \delta$ and $\eta$ on stability. The objective of this section is to illustrate how $N, \eta$ and $\delta$ all impact each other. Our numerical study illustrates that for a given $\delta$, we can chose one of several potential interval lengths $N$, by using the appropriate $\eta$. This demonstrates the flexibility of our approach.

In particular, we first fix values for $\delta$ and we study the smallest $\eta$ such that (20)-(22) hold for a given $N$. Fixing $\delta$ is relevant from a practical point of view as the maximum packet success rate is determined by the wireless network. We plot the smallest $\eta$ which results in MSS in Figure 3 against $N$ for certain values of $\delta$. Surprisingly, we observe that even for very low maximum packet success rates, i.e. $\delta=0.2$, we are able to achieve MSS with a large enough $\eta$. That is, if the probability for the transmission interval to be beyond $N$ is small enough, the system is MSS. We also note that the $\delta$-SATI for $\delta=0.5$ is lower bounded by 26 and the $\delta$-SATI for $\delta=0.2$ is lower bounded by 19 .

Next, we demonstrate the flexibility in choosing $\delta$ and $\eta$, by presenting the image of the lower bound on the corresponding $(\eta, \delta)$-SATI in Figure 4. In practice $\delta$ might be determined by the wireless network, however the flexibility in choosing $\eta$ and the corresponding $(\eta, \delta)$-SATI allows us to select the right configuration for designing the optimal communication scheme based on the communication system requirements. For example, if every transmission attempt costs the same amount of energy, transmitting periodically, with a period length of the $\delta$-SATI interval might be the most efficient, as no energy is consumed within this interval. On the other hand, if the communication cost depends on the 


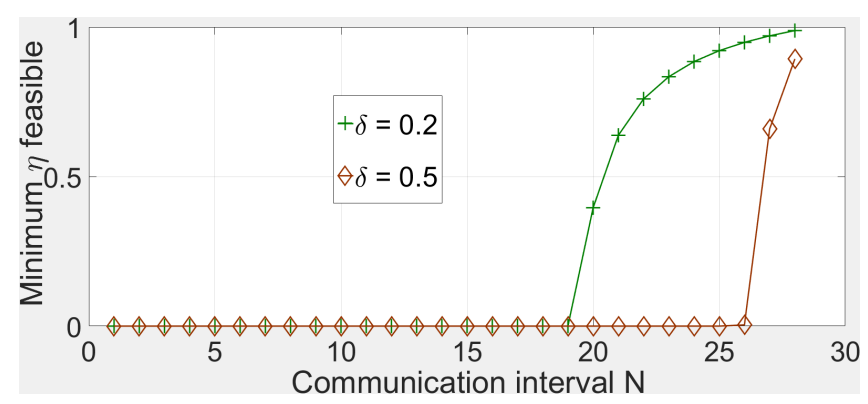

Fig. 3: The smallest feasible $\eta$ plotted against $N$. The largest value of $N$ feasible for a given $\eta$ and $\delta$ corresponds to the $(\eta, \delta)$-SATI.

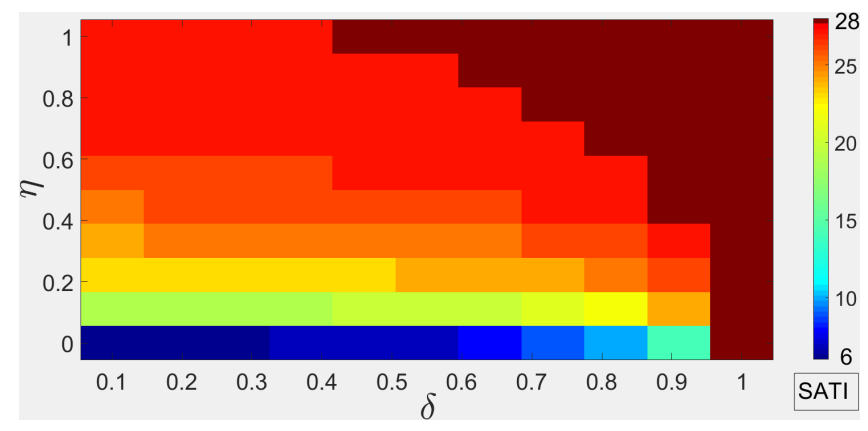

Fig. 4: The $(\eta, \delta)$-SATI (lower bounds) for various values of $\delta$ and $\eta$. The color indicates the value of the $(\eta, \delta)$-SATI, and we notice that even when $\delta=0.1$ and $\eta=0$, a $\delta$-SATI of 6 is possible.

time-varying strength of the wireless channel, selecting a larger $\eta$ might be more suitable. This enables us to select a longer $(\eta, \delta)$-SATI, as long as communications are attempted even within the interval to meet the constraint (8) based on $\eta$. Communication within this interval can be optimized based on the wireless channel as we have done in [19] for the deterministic setting.

\section{CONCLUSION}

We have studied discrete-time linear systems in which the communication with the controller occurs over a stochastic wireless channel. We introduce the notion of $(\eta, \delta)$-SATI which is an interpretation of the traditional MATI in the stochastic setting, where $\eta$ denotes the cumulative probability of a successful transmission within the $(\eta, \delta)$-SATI and $\delta$ denotes the instantaneous communication success probability after the $(\eta, \delta)$-SATI has passed. Under the assumption that the system with perfect communication is stabilizable, we provide conditions on the $(\eta, \delta)$-SATI in terms of matrix inequalities. The obtained results will be exploited in future work for the design of energy-efficient communication strategies depending on the channel fading, while ensuring the MSS of the WNCS. Hence, extending our previous work [19] which considered a MATI constraint.

\section{ACKNOWLEDGMENT}

We would like thank Alejandro. I. Maass for helpful discussions.

\section{REFERENCES}

[1] D. Carnevale, A.R. Teel, and D. Nešić. A Lyapunov proof of an improved maximum allowable transfer interval for networked control systems. IEEE Trans. on Automatic Control, 52(5):892-897, 2007.

[2] V.S. Dolk and W.P.M.H. Heemels. Dynamic event-triggered control under packet losses: The case with acknowledgements. In Eventbased Control, Communication, and Signal Processing (EBCCSP), 2015 International Conference on, pages 1-7. IEEE, 2015.

[3] M.C.F. Donkers, W.P.M.H. Heemels, D. Bernardini, A. Bemporad, and V. Shneer. Stability analysis of stochastic networked control systems. Automatica, 48(5):917-925, 2012.

[4] M.C.F. Donkers, W.P.M.H. Heemels, N. van de Wouw, and L. Hetel. Stability analysis of networked control systems using a switched linear systems approach. IEEE Transactions on Automatic Control, 56(9):2101-2115, 2011.

[5] L. E. Ghaoui and M. A. Rami. Robust state-feedback stabilization of jump linear systems via LMIs. International Journal of Robust and Nonlinear Control, 6(910):1015-1022, 1996.

[6] W.P.M.H. Heemels, A.R. Teel, N. van de Wouw, and D. Nešić. Networked control systems with communication constraints: Tradeoffs between transmission intervals, delays and performance. IEEE Trans. on Aut. Control, 55(8):1781-1796, 2010.

[7] W.P.M.H. Heemels and N. van de Wouw. Stability and stabilization of networked control systems. In Networked Control Systems, volume Volume 406 of the series Lecture Notes in Control and Information Sciences, pages 203-253. Springer, 2010.

[8] J. Hespanha, P. Naghshtabrizi, and Y. Xu. A survey of recent results in networked control systems. IEEE Special Issue on Technology of Networked Control Systems, 95(1):138-162, 2007.

[9] A. Jentzen, F. Leber, D. Schneisgen, A. Berger, and S. Siegmund. An improved maximum allowable transfer interval for $\mathcal{L}_{p}$-stability of networked control systems. IEEE Transactions on Automatic Control, 55(1):179-184, 2010.

[10] L.A. Montestruque and P. Antsaklis. Stability of model-based networked control systems with time-varying transmission times. IEEE Transactions on Automatic Control, 49(9):1562-1572, 2004.

[11] D. Nešić and A.R. Teel. Input-output stability properties of networked control systems. IEEE Trans. on Aut. Control, 49:1650-1667, 2004.

[12] H. Omran, L. Hetel, J.-P. Richard, and F. Lamnabhi-Lagarrigue. Stability analysis of bilinear systems under aperiodic sampled-data control. Automatica, 50(4):1288-1295, 2014.

[13] R. Postoyan and D. Nešić. Time-triggered control of nonlinear discrete-time systems. In IEEE Conference on Decision and Control, Las Vegas, U.S.A., pages 6814-6819, 2016.

[14] R. Postoyan and D. Nešić. A framework for the observer design for networked control systems. IEEE Transactions on Automatic Control, 57(5):1309-1314, 2012.

[15] R. Postoyan, N. van de Wouw, D. Nešić, and W.P.M.H. Heemels. Tracking control for nonlinear networked control systems. IEEE Transactions on Automatic Control, 59(6):1539-1554, 2014.

[16] L. Schenato. To zero or to hold control inputs with lossy links? IEEE Transactions on Automatic Control, 54(5):1093-1099, 2009.

[17] A. Sheth, S. Nedevschi, R. Patra, S. Surana, E. Brewer, and L. Subramanian. Packet loss characterization in wifi-based long distance networks. In INFOCOM 2007. 26th IEEE International Conference on Computer Communications. IEEE, pages 312-320. IEEE, 2007.

[18] S. R. Theodore. Wireless communications: principles and practice, volume 2. Prentice Hall PTR New Jersey, 1996.

[19] V.S. Varma and R. Postoyan. Energy efficient time-triggered control over wireless sensor/actuator networks. In IEEE Conference on Decision and Control, Las Vegas, U.S.A., 2016.

[20] L. Xie and L. Xie. Stabilizing sampled-data linear systems with markovian packet losses and random sampling. IFAC Proceedings Volumes, 41(2):599-604, 2008.

[21] W. Zhang and M.S. Branicky. Stability of networked control systems with time-varying transmission period. In Allerton Conference on Communication, Control, and Computing, 2001.

[22] J. Zhao and R. Govindan. Understanding packet delivery performance in dense wireless sensor networks. In Proceedings of the 1st international conference on Embedded networked sensor systems, pages 1-13. ACM, 2003. 